\title{
The inadequacy of linear autoregressive model for real exchange rates: empirical evidence from Asian economies
}

\begin{abstract}
Utilizing the formal linearity test of Luukkonen, Saikkonen and Teräsvirta (Biometrika, 75, 491-499, 1998) as diagnostic tool, the empirical finding suggests that the linear autoregressive (AR) model is inadequate in describing the real exchange rates behaviour of 11 Asian economies. It is noted that the conventional battery of diagnostic tests is capable of identifying the inadequacy of the linear model in only three of these series. Moreover, the linearity nature of this behaviour has been formally rejected in favour of the non-linear smooth transition autoregressive (STAR) model. The finding of non-linearity in the data generating process of these real exchange rates warrants that the use of linear framework in empirical modelling and statistical testing procedures in the field of exchange rates may lead to an inappropriate policy conclusions.
\end{abstract}

Keyword: Real exchange rates; Asian economies 